\title{
Bilinear approach to soliton and periodic wave solutions of two nonlinear evolution equations of Mathematical Physics
}

\author{
Rui Cao ${ }^{1 *}$, Qiulan Zhao ${ }^{2}$ and Lin $\mathrm{Gao}^{3}$
}

"Correspondence:

ruicao999@126.com

${ }^{1}$ College of Mathematics and

Statistics, Heze University, Heze, P.R.

China

Full list of author information is

available at the end of the article

\section{Springer}

\begin{abstract}
In the present paper, the potential Kadomtsev-Petviashvili equation and $(3+1)$-dimensional potential-YTSF equation are investigated, which can be used to describe many mathematical and physical backgrounds, e.g., fluid dynamics and communications. Based on Hirota bilinear method, the bilinear equation for the $(3+1)$-dimensional potential-YTSF equation is obtained by applying an appropriate dependent variable transformation. Then $\mathrm{N}$-soliton solutions of nonlinear evolution equation are derived by the perturbation technique, and the periodic wave solutions for potential Kadomtsev-Petviashvili equation and $(3+1)$-dimensional potential-YTSF equation are constructed by employing the Riemann theta function. Furthermore, the asymptotic properties of periodic wave solutions show that soliton solutions can be derived from periodic wave solutions.
\end{abstract}

Keywords: Potential Kadomtsev-Petviashvili equation; $(3+1)$-dimensional potential-YTSF equation; $\mathrm{N}$-soliton solution; Periodic wave solution; Hirota method

\section{Introduction}

The construction of analytic solutions for nonlinear evolution equations (NLEEs) is a key topic in the study of nonlinear phenomena [1-7]. Analytic solutions can help one to well understand the mechanism of physical phenomena modeled by a nonlinear evolution equation. With the development of soliton theory and computer algebraic system like Maple, much attention has been paid to finding analytic solutions of nonlinear evolution equations, including soliton solutions, periodic wave solutions, shock wave solutions, and so on. Up to now, many powerful methods of searching for exact solutions to NLEEs have been proposed and developed. For example, Biswas and Bhrawy [8] employed the extended Jacobi elliptic function expansion method to study the Zakharov equation and the Davey-Stewartson equation and obtained cnoidal and snoidal wavesolutions. Ma and Lee et al. [9] investigated a $3+1$ dimensional Jimbo-Miwa equation via a transformed rational function method and obtained exact solutions. Other methods, such as Bäcklund transformation [10,11], Darboux transformation [12, 13], Hirota bilinear method [14], the inverse scattering transform, the variable separation method [15], the sine-cosine method, the tanh-function method [16], the auxiliary equation method [17], the trial function method [18], etc., were also employed. Based on these methods, a variety of nonlinear equations have been investigated and solved.

(c) The Author(s) 2019. This article is distributed under the terms of the Creative Commons Attribution 4.0 International License (http://creativecommons.org/licenses/by/4.0/), which permits unrestricted use, distribution, and reproduction in any medium, provided you give appropriate credit to the original author(s) and the source, provide a link to the Creative Commons license, and indicate if changes were made. 
Among them, the Hirota method is one of the most effective methods of constructing multiple soliton solutions of NLEEs. It can transform the given nonlinear evolution equations to the corresponding bilinear forms through the dependent variable transformation. Then employing the perturbation expansion method, multi-soliton solutions with exponential function are derived. Also through the bilinear Bäcklund transformation, Lax pairs are obtained. In recent years, the Hirota method has been developed to construct the Wronskian solutions, Pfaffian solutions, and periodic wave solutions by use of the Riemann theta functions [19-24]. By means of this method, Tian et al. have investigated the HS equation for shallow water waves and BLMP equation [19]. And Ma, Zhang et al. have constructed periodic wave solutions of $(2+1)$-dimensional Hirota bilinear equations and Ito equation [20,21]. The advantage of this method lies in the fact that we obtain the periodic wave solutions in a direct method without algebraic-geometric theory. Furthermore, the soliton solutions can be derived from the periodic wave solutions via asymptotic analysis.

With a motivation to further expand the area of applications of this method, in the present paper, we study the potential Kadomtsev-Petviashvili equation and $(3+1)$ dimensional potential-YTSF equation to illustrate the efficiency of using the combination of the Hirota method and the Riemann theta function. To the best of our knowledge, these results are up to date and have not been reported.

The rest of the paper is organized as follows. In Sect. 2, the bilinear form of $(3+1)$ dimensional potential-YTSF equation is given by applying the Hirota bilinear method. In Sect. 3, N-soliton solutions are presented by using the perturbation approach. In Sect. 4, by virtue of the Riemann theta function, periodic wave solutions are derived successfully, and the asymptotic properties of periodic wave solutions show that periodic wave solution degenerate to soliton solution. Finally, the concluding remarks are presented in Sect. 5.

\section{Bilinear form for $(3+1)$-dimensional potential-YTSF equation}

In this section, we will give the bilinear form for the $(3+1)$-dimensional potential-YTSF equation by applying the Hirota direct method and the dependent variable transformation.

A new $(3+1)$-dimensional nonlinear evolution equation, called the potential YTSF equation, was first introduced by Yu, Toda, Sasa and Fukuyama (YTSF) [25]. The (3+1)dimensional potential-YTSF equation can be written as

$$
-4 u_{x t}+u_{x x x z}+3 u_{x} u_{x z}+3 u_{x x} u_{z}+3 u_{y y}=0 .
$$

Setting $u=\frac{3}{4} w_{x}$, substituting it into Eq. (2.1) and integrating with respect to $x$ yields

$$
-4 w_{x t}+w_{x x x z}+3 w_{x x} w_{x z}+3 w_{y y}+\lambda=0
$$

which is transformed into the bilinear representation

$$
\left[-4 D_{x} D_{t}+D_{x}^{3} D_{z}+3 D_{y}^{2}\right] F \cdot F+\lambda F^{2}=0,
$$

under the dependent variable transformation $w=2 \ln F$, where $\lambda=\lambda(y, z, t)$ is an integration constant; $D_{x}, D_{y}$ and $D_{t}$ are the well-known Hirota operators defined by [21]

$$
D_{x}^{m} D_{t}^{n} f \cdot g=\left(\partial_{x}-\partial_{x^{\prime}}\right)^{m}\left(\partial_{t}-\partial_{t^{\prime}}\right)^{n} f(x, t) \times\left. g\left(x^{\prime}, t^{\prime}\right)\right|_{x^{\prime}=x, t^{\prime}=t} .
$$


The $D$-operators have the following nice property when acting on exponential functions:

$$
D_{x}^{m} D_{y}^{s} D_{t}^{n} \exp \xi_{1} \cdot \exp \xi_{2}=\left(P_{1}-P_{2}\right)^{m}\left(Q_{1}-Q_{2}\right)^{s}\left(\Omega_{1}-\Omega_{2}\right)^{n} \exp \left(\xi_{1}+\xi_{2}\right)
$$

where $\xi_{i}=P_{i} x+Q_{i} y+\Omega_{i} t+\xi_{i}^{0}(i=1,2)$.

More generally, we get

$$
G\left(D_{x}, D_{y}, D_{t}\right) \exp \xi_{1} \cdot \exp \xi_{2}=G\left(P_{1}-P_{2}, Q_{1}-Q_{2}, \Omega_{1}-\Omega_{2}\right) \exp \left(\xi_{1}+\xi_{2}\right)
$$

Remark $1 D$ operates on a product of two functions like the Leibniz rule, except for a crucial sign difference. For example,

$$
\begin{aligned}
& D_{x} G \cdot F=G_{x} F-G F_{x}, \\
& D_{x} D_{t} G \cdot F=G_{x t} F-G_{x} F_{t}+G F_{x t}-G_{t} F_{x}, \\
& D_{x x} G \cdot F=G_{x x} F-2 G_{x} F_{x}+G F_{x x} .
\end{aligned}
$$

\section{$3 \mathbf{N}$-soliton solutions for ( $3+1)$-dimensional potential-YTSF equation}

In the following, we will give $\mathrm{N}$-soliton solutions for the $(3+1)$-dimensional potentialYTSF equation by virtue of the Hirota method and the perturbation expansion and truncation technique, as well as property (2.5).

Expanding $F$ into the power series with respect to a small parameter $\varepsilon$ gives

$$
F=1+\varepsilon f_{1}+\varepsilon^{2} f_{2}+\varepsilon^{3} f_{3}+\cdots .
$$

Substituting (3.1) into bilinear equation (2.3) and setting the coefficients of the same power of $\varepsilon$ to zero, we obtain the recursion relations for $f_{i}$.

Single-soliton solution For $n=1$, Eq. (3.1) becomes

$$
f_{1}=\exp \left(\eta_{1}\right), \quad \eta_{1}=k_{1} x+l_{1} y+m_{1} z+\omega_{1} t+\eta_{1}^{0}
$$

where $k_{1}, l_{1}, m_{1}, \omega_{1}$ are arbitrary constants, and $-4 k_{1} \omega_{1}+k_{1}^{3} m_{1}+3 l_{1}^{2}=0$ is the dispersion relation.

Substituting (3.2) into bilinear equation (2.3), a single-soliton solution of Eq. (2.1) is given by

$$
u(x, y, z, t)=\frac{3}{2}\left[\ln \left(1+e^{\eta_{1}}\right)\right]_{x} .
$$

Two-soliton solution A two-soliton solution is given by

$$
u(x, y, z, t)=\frac{3}{2}\left[\ln \left(1+e^{\eta_{1}}+e^{\eta_{2}}+e^{\eta_{1}+\eta_{2}+A_{12}}\right)\right]_{x^{\prime}}
$$

where $-4 k_{i} \omega_{i}+k_{i}^{3} m_{i}+3 l_{i}^{2}=0(i=1,2)$ are the dispersion relations and the phase shift term is

$$
e^{A_{12}}=-\frac{4\left(k_{1}-k_{2}\right)\left(\omega_{1}-\omega_{2}\right)-\left(k_{1}-k_{2}\right)^{3}\left(m_{1}-m_{2}\right)-3\left(l_{1}-l_{2}\right)^{2}}{4\left(k_{1}+k_{2}\right)\left(\omega_{1}+\omega_{2}\right)-\left(k_{1}+k_{2}\right)^{3}\left(m_{1}+m_{2}\right)-3\left(l_{1}+l_{2}\right)^{2}} .
$$


N-soliton solution Now we derive N-soliton solutions as

$$
u(x, y, z, t)=\frac{3}{2}\left[\ln \sum_{\mu=0,1} e^{\sum_{i=1}^{N} \mu_{i} \eta_{i}+\sum_{i<j}^{(N)} \mu_{i} \mu_{j} A_{i j}}\right]_{x}, \quad 1 \leq i, j \leq N
$$

where the phase shift term is

$$
e^{A_{i j}}=-\frac{4\left(k_{i}-k_{j}\right)\left(\omega_{i}-\omega_{j}\right)-\left(k_{i}-k_{j}\right)^{3}\left(m_{i}-m_{i}\right)-3\left(l_{i}-l_{j}\right)^{2}}{4\left(k_{i}+k_{j}\right)\left(\omega_{i}+\omega_{j}\right)-\left(k_{i}+k_{j}\right)^{3}\left(m_{i}+m_{j}\right)-3\left(l_{i}+l_{j}\right)^{2}} .
$$

Here $\sum_{\mu=0,1}$ means a summation over all possible combinations of $\mu_{j}=0,1(j=1,2, \ldots, N)$ and $\sum_{i<j}^{N}$ is a summation over all possible pairs $(i, j)(i=1, \ldots, N, j=1, \ldots, N)$ with the condition that $i<j$.

\section{Periodic wave solutions of two equations}

In this section, we will construct periodic wave solutions for the potential KadomtsevPetviashvili equation and the $(3+1)$-dimensional potential-YTSF equation by employing the Hirota method and the Riemann theta function, as well as property (2.5).

\subsection{Potential Kadomtsev-Petviashvili equation}

The following $(2+1)$-dimensional potential Kadomtsev-Petviashvili equation [26] is considered:

$$
u_{t}+\frac{3}{4} u_{x}^{2}+\frac{1}{4} u_{x x x}+\frac{3}{4} \partial_{x}^{-1} u_{y y}=0
$$

which is transformed into the bilinear form

$$
\left[4 D_{x} D_{t}+D_{x}^{4}+3 D_{y}^{2}\right] F \cdot F=0
$$

under the dependent variable transformation $u=2(\ln F)_{x}$.

We introduce the Riemann theta function solution of Eq. (4.1) as

$$
F=\sum_{n=-\infty}^{\infty} e^{2 \pi i n \zeta+\pi i n^{2} \tau}
$$

where $n \in \mathcal{Z}, \tau \in \mathcal{C}, \operatorname{Im} \tau>0$ and $\zeta=k x+l y+\omega t$.

Substituting (4.3) into (4.2), we get

$$
\begin{aligned}
G & \left(D_{x}, D_{y}, D_{t}\right) F \cdot F \\
& =G\left(D_{x}, D_{y}, D_{t}\right) \sum_{n=-\infty}^{\infty} e^{2 \pi i n \zeta+\pi i n^{2} \tau} \sum_{m=-\infty}^{\infty} e^{2 \pi i m \zeta+\pi i m^{2} \tau} \\
& =\sum_{n=-\infty}^{\infty} \sum_{m=-\infty}^{\infty} G\left(D_{x}, D_{y}, D_{t}\right) e^{2 \pi i n \zeta+\pi i n^{2} \tau} e^{2 \pi i m \zeta+\pi i m^{2} \tau} \\
& =\sum_{n=-\infty}^{\infty} \sum_{m=-\infty}^{\infty} G[2 \pi i(n-m) k, 2 \pi i(n-m) l, 2 \pi i(n-m) \omega] e^{2 \pi i(n+m) \zeta+\pi i\left(n^{2}+m^{2}\right) \tau}
\end{aligned}
$$




$$
\begin{aligned}
& =\sum_{p=-\infty}^{\infty} \sum_{n=-\infty}^{\infty} G[2 \pi i(2 n-p) k, 2 \pi i(2 n-p) l, 2 \pi i(2 n-p) \omega] e^{\pi i\left(n^{2}+(p-n)^{2}\right) \tau} e^{2 \pi i p \zeta} \\
& =\sum_{p=-\infty}^{\infty} \bar{G}(p) e^{2 \pi i p \zeta}
\end{aligned}
$$

where $n+m=p$. Noting that

$$
\begin{aligned}
\bar{G}(p)= & \sum_{n=-\infty}^{\infty} G(2 \pi i(2 n-p) k, 2 \pi i(2 n-p) l, 2 \pi i(2 n-p) \omega] e^{\pi i\left(n^{2}+(p-n)^{2}\right) \tau} \\
= & \left.\sum_{N=-\infty}^{\infty} G[2 \pi i(2 N-(p-2)) k, 2 \pi i(2 N-(p-2)) l, 2 \pi i \times(2 N-(p-2)) \omega)\right] \\
& \times e^{\pi i\left((N+1)^{2}+(p-N-1)^{2}\right) \tau} \\
= & \left.\sum_{N=-\infty}^{\infty} G[2 \pi i(2 N-(p-2)) k, 2 \pi i(2 N-(p-2)) l, 2 \pi i(2 N-(p-2)) \omega)\right] \\
& \times e^{\pi i\left(N^{2}+(p-N-2)^{2}\right) \tau} e^{2 \pi i(p-1) \tau} \\
= & \bar{G}(p-2) e^{2 \pi i(p-1) \tau},
\end{aligned}
$$

which indicates that if $\bar{G}(0)=\bar{G}(1)=0$, then

$$
\bar{G}(p)=0, \quad p \in \mathcal{Z} .
$$

Therefore, we may let

$$
\begin{aligned}
\bar{G}(0)= & \sum_{n=-\infty}^{\infty}\left[-64 \pi^{2} n^{2} k \omega+256 \pi^{4} n^{4} k^{4}-48 \pi^{2} n^{2} l^{2}+\mu\right] e^{2 \pi i n^{2} \tau}=0, \\
\bar{G}(1)= & \sum_{n=-\infty}^{\infty}\left[-16 \pi^{2}(2 n-1)^{2} k \omega+16 \pi^{4}(2 n-1)^{4} k^{4}-12 \pi^{2}(2 n-1)^{2} l^{2}+\mu\right] \\
& \times e^{\pi i\left(n^{2}+(n-1)^{2}\right) \tau}=0 .
\end{aligned}
$$

Denote

$$
\begin{aligned}
& \Delta_{1}(n)=e^{2 \pi i n^{2} \tau}, \quad \Delta_{2}(n)=e^{\pi i\left(n^{2}+(n-1)^{2}\right) \tau}, \\
& A_{11}=-\sum_{n=-\infty}^{\infty} 64 \pi^{2} n^{2} k \Delta_{1}(n), \quad A_{12}=\sum_{n=-\infty}^{\infty} \Delta_{1}(n), \\
& A_{21}=-\sum_{n=-\infty}^{\infty} 16 \pi^{2}(2 n-1)^{2} k \Delta_{2}(n), \quad A_{22}=\sum_{n=-\infty}^{\infty} \Delta_{2}(n), \\
& B_{1}=-\sum_{n=-\infty}^{\infty}\left(256 \pi^{4} n^{4} k^{4}-48 \pi^{2} n^{2} l^{2}\right) \Delta_{1}(n), \\
& B_{2}=-\sum_{n=-\infty}^{\infty}\left[16 \pi^{4}(2 n-1)^{4} k^{4}-12 \pi^{2}(2 n-1)^{2} l^{2}\right] \Delta_{2}(n) .
\end{aligned}
$$


Then Eqs. (4.5) and (4.6) can be written as

$$
A_{11} \omega+A_{12} \mu=B_{1}, \quad A_{21} \omega+A_{22} \mu=B_{2} .
$$

By solving this system, we get

$$
\omega=\frac{B_{1} A_{22}-A_{12} B_{2}}{A_{11} A_{22}-A_{12} A_{21}}, \quad \mu=\frac{A_{11} B_{2}-A_{21} B_{1}}{A_{11} A_{22}-A_{12} A_{21}} .
$$

Thus the periodic wave solution is given by

$$
u=2(\ln F)_{x}
$$

where $\omega$ and $F$ are determined by Eqs. (4.7) and (4.3), respectively.

Next, we will demonstrate that the soliton solution can be obtained as a limit of a periodic wave solution. From Eq. (4.3), we rewrite $F$ as

$$
F=1+\alpha\left(e^{2 \pi i \zeta}+e^{-2 \pi i \zeta}\right)+\alpha^{4}\left(e^{4 \pi i \zeta}+e^{-4 \pi i \zeta}\right)+\cdots,
$$

where $\alpha=e^{\pi i \tau}$.

Setting

$$
K=2 \pi i k, \quad L=2 \pi i l, \quad \Omega=2 \pi i \omega, \quad \zeta^{\prime}=K x+L y+\Omega t+\pi i \tau,
$$

we get

$$
\begin{aligned}
F & =1+\alpha\left(e^{2 \pi i \zeta}+e^{-2 \pi i \zeta}\right)+\alpha^{4}\left(e^{4 \pi i \zeta}+e^{-4 \pi i \zeta}\right)+\cdots \\
& =1+e^{\zeta^{\prime}}+\alpha^{2}\left(e^{-\zeta^{\prime}}+e^{2 \zeta^{\prime}}\right)+\alpha^{6}\left(e^{-2 \zeta^{\prime}}+e^{3 \zeta^{\prime}}\right)+\cdots \\
& \rightarrow 1+e^{\zeta^{\prime}}, \quad \text { as } \alpha \rightarrow 0 .
\end{aligned}
$$

Thus, the periodic wave solution (4.8) turns to the soliton solution

$$
u=2(\ln F)_{x}, \quad F=1+e^{\zeta^{\prime}}, \quad \zeta^{\prime}=K x+L y+\Omega t+\pi i \tau,
$$

if we can prove that

$$
\Omega \rightarrow-\frac{K^{3}}{4}-\frac{3 L^{2}}{4 K}
$$

In fact, it is easy to known that

$$
\begin{aligned}
& A_{11}=-128 \pi^{2} k\left(\alpha^{2}+4 \alpha^{8}+\cdots\right), \quad A_{12}=1+2 \alpha^{2}+2 \alpha^{8}+\cdots, \\
& A_{21}=-32 \pi^{2} k\left(\alpha+9 \alpha^{5}+\cdots\right), \quad A_{22}=2 \alpha+2 \alpha^{5}+\cdots, \\
& B_{1}=2\left(256 \pi^{4} k^{4}-48 \pi^{2} l^{2}\right) \alpha^{2}+2\left(256 \pi^{4} 2^{4} k^{4}-48 \pi^{2} 2^{2} l^{2}\right) \alpha^{8}+\cdots, \\
& B_{2}=-2\left(16 \pi^{4} k^{4}-12 \pi^{2} l^{2}\right) \alpha+2\left(16 \pi^{4} 3^{4} k^{4}-12 \pi^{2} 3^{2} l^{2}\right) \alpha^{5}+\cdots,
\end{aligned}
$$


which lead to

$$
\begin{aligned}
& B_{1} A_{22}-A_{12} B_{2}=2\left(16 \pi^{4} k^{4}-12 \pi^{2} l^{2}\right) \alpha+o(\alpha), \\
& A_{11} A_{22}-A_{12} A_{21}=32 \pi^{2} k \alpha+o(\alpha),
\end{aligned}
$$

According to (4.7), we get

$$
\omega \rightarrow \pi^{2} k^{3}-\frac{3 l^{2}}{4 k}, \quad \text { as } \alpha \rightarrow 0,
$$

which is equivalent to

$$
\Omega \rightarrow-\frac{K^{3}}{4}-\frac{3 L^{2}}{4 K}, \quad \text { as } \alpha \rightarrow 0 .
$$

\section{$4.2(3+1)$-dimensional potential-YTSF equation}

By a similar analysis process as in Sect. 4.1, we have

$$
\begin{aligned}
\bar{G}(0)= & \sum_{n=-\infty}^{\infty}\left[64 \pi^{2} n^{2} k \omega+256 \pi^{4} n^{4} k^{3} m-48 \pi^{2} n^{2} l^{2}+\lambda\right] e^{2 \pi i n^{2} \tau}=0, \\
\bar{G}(1)= & \sum_{n=-\infty}^{\infty}\left[16 \pi^{2}(2 n-1)^{2} k \omega+16 \pi^{4}(2 n-1)^{4} k^{3} m-12 \pi^{2}(2 n-1)^{2} l^{2}+\lambda\right] \\
& \times e^{\pi i\left(n^{2}+(n-1)^{2}\right) \tau}=0 .
\end{aligned}
$$

Denote

$$
\begin{aligned}
& \Delta_{1}(n)=e^{2 \pi i n^{2} \tau}, \quad \Delta_{2}(n)=e^{\pi i\left(n^{2}+(n-1)^{2}\right) \tau}, \\
& A_{11}=\sum_{n=-\infty}^{\infty} 64 \pi^{2} n^{2} k \Delta_{1}(n), \quad A_{12}=\sum_{n=-\infty}^{\infty} \Delta_{1}(n), \\
& A_{21}=\sum_{n=-\infty}^{\infty} 16 \pi^{2}(2 n-1)^{2} k \Delta_{2}(n), \quad A_{22}=\sum_{n=-\infty}^{\infty} \Delta_{2}(n), \\
& B_{1}=-\sum_{n=-\infty}^{\infty}\left(256 \pi^{4} n^{4} k^{3} m-48 \pi^{2} n^{2} l^{2}\right) \Delta_{1}(n), \\
& B_{2}=-\sum_{n=-\infty}^{\infty}\left[16 \pi^{4}(2 n-1)^{4} k^{3} m-12 \pi^{2}(2 n-1)^{2} l^{2}\right] \Delta_{2}(n) .
\end{aligned}
$$

Then Eqs. (4.15) and (4.16) can be written as

$$
A_{11} \omega+A_{12} \lambda=B_{1}, \quad A_{21} \omega+A_{22} \lambda=B_{2} .
$$

By solving the system, we get

$$
\omega=\frac{B_{1} A_{22}-A_{12} B_{2}}{A_{11} A_{22}-A_{12} A_{21}}, \quad \lambda=\frac{A_{11} B_{2}-A_{21} B_{1}}{A_{11} A_{22}-A_{12} A_{21}} .
$$


Thus, the periodic wave solution is given by

$$
u=\frac{3}{2}(\ln F)_{x}
$$

where $\omega$ and $F$ are determined by Eqs. (4.17) and (4.3), respectively.

From Eq. (4.3), we rewrite $F$ as

$$
F=1+\delta\left(e^{2 \pi i \zeta}+e^{-2 \pi i \zeta}\right)+\delta^{4}\left(e^{4 \pi i \zeta}+e^{-4 \pi i \zeta}\right)+\cdots,
$$

where $\delta=e^{\pi i \tau}$.

Setting

$$
\begin{aligned}
& K=2 \pi i k, \quad L=2 \pi i l, \quad M=2 \pi i m, \quad \Omega=2 \pi i \omega, \\
& \zeta^{\prime}=K x+L y+M z+\Omega t+\pi i \tau,
\end{aligned}
$$

yields

$$
\begin{aligned}
F & =1+\delta\left(e^{2 \pi i \zeta}+e^{-2 \pi i \zeta}\right)+\delta^{4}\left(e^{4 \pi i \zeta}+e^{-4 \pi i \zeta}\right)+\cdots \\
& =1+e^{\zeta^{\prime}}+\delta^{2}\left(e^{-\zeta^{\prime}}+e^{2 \zeta^{\prime}}\right)+\delta^{6}\left(e^{-2 \zeta^{\prime}}+e^{3 \zeta^{\prime}}\right)+\cdots \\
& \rightarrow 1+e^{\zeta^{\prime}}, \quad \text { as } \delta \rightarrow 0 .
\end{aligned}
$$

Thus, if we can prove that

$$
\Omega \rightarrow \frac{K^{2} M}{4}+\frac{3 L^{2}}{4 K}, \quad \text { as } \delta \rightarrow 0,
$$

the periodic wave solution (4.18) turns to the soliton solution

$$
u=\frac{3}{2}(\ln F)_{x}, \quad F=1+e^{\zeta^{\prime}}, \quad \zeta^{\prime}=K x+L y+M z+\Omega t+\pi i \tau .
$$

In fact, it is easy to known that

$$
\begin{aligned}
& A_{11}=128 \pi^{2} k\left(\delta^{2}+4 \delta^{8}+\cdots\right), \\
& A_{12}=1+2 \delta^{2}+2 \delta^{8}+\cdots, \quad A_{21}=32 \pi^{2} k\left(\delta+9 \delta^{5}+\cdots\right), \quad A_{22}=2 \delta+2 \delta^{5}+\cdots, \\
& B_{1}=2\left(256 \pi^{4} k^{3} m-48 \pi^{2} l^{2}\right) \delta^{2}+2\left(256 \pi^{4} 2^{4} k^{3} m-48 \pi^{2} 2^{2} l^{2}\right) \delta^{8}+\cdots, \\
& B_{2}=-2\left(16 \pi^{4} k^{3} m-12 \pi^{2} l^{2}\right) \delta+2\left(16 \pi^{4} 3^{4} k^{3} m-12 \pi^{2} 3^{2} l^{2}\right) \delta^{5}+\cdots,
\end{aligned}
$$

which lead to

$$
\begin{aligned}
& B_{1} A_{22}-A_{12} B_{2}=2\left(16 \pi^{4} k^{3} m-12 \pi^{2} l^{2}\right) \delta+o(\delta), \\
& A_{11} A_{22}-A_{12} A_{21}=-32 \pi^{2} k \delta+o(\delta) .
\end{aligned}
$$

According to (4.17), we get

$$
\omega \rightarrow-\pi^{2} k^{2} m+\frac{3 l^{2}}{4 k}, \quad \text { as } \delta \rightarrow 0,
$$


which is equivalent to

$$
\Omega \rightarrow \frac{K^{2} M}{4}+\frac{3 L^{2}}{4 K}, \quad \text { as } \delta \rightarrow 0 .
$$

\section{Discussion and conclusion}

In the present paper, we investigate the $(2+1)$-dimensional potential KP equation and $(3+$ 1)-dimensional potential-YTSF equation based on the Hirota method and the Riemann theta function. As a result, we obtain the bilinear form and $\mathrm{N}$-soliton solutions of the $(3+1)$-dimensional potential-YTSF equation under constraint conditions. By virtue of the Hirota method and the Riemann theta function, periodic wave solutions have been presented. And via asymptotic analysis, classical soliton solutions have been derived from their periodic wave solutions. Finally, it is worthwhile to note that the Hirota direct method can be applied to other variable coefficient NLEEs in mathematical physics.

\section{Acknowledgements}

The authors are very grateful to the editor and the referees for their insightful and constructive comments and suggestions, which have led to an improved version of this paper.

\section{Funding}

This work is supported in part by the NNSF of China (No. 11701334, 11347102), the Natural Science Foundation of Shandong Province (ZR2014AM032), the project of Shandong Province Higher Educational Science and Technology Program (J16LI15) and the Fund Project of Heze University (XY16BS12, XY18KJ09).

\section{Competing interests}

The authors declare that there is no conflict of interest regarding the publication of this paper

\section{Authors' contributions}

The authors carried out the calculation and conceived of the study. The authors read and approved the final manuscript.

\section{Author details}

${ }^{1}$ College of Mathematics and Statistics, Heze University, Heze, P.R. China. ${ }^{2}$ College of Mathematics and Systems Science, Shandong University of Science and Technology, Qingdao, P.R. China. ${ }^{3}$ College Library, Heze University, Heze, P.R. China.

\section{Publisher's Note}

Springer Nature remains neutral with regard to jurisdictional claims in published maps and institutional affiliations.

Received: 14 December 2018 Accepted: 4 March 2019 Published online: 25 April 2019

\section{References}

1. Lv, X., Ma, W.X., Khalique, C.M.: A direct bilinear Bäcklund transformation of a $(2+1)$-dimensional Korteweg-de Vries-like model. Appl. Math. Lett. 50, 37-42 (2015)

2. Lu, X., Chen, S.T., Ma, W.X.: Constructing lump solutions to a generalized Kadomtsev-Petviashvili-Boussinesq equation. Nonlinear Dyn. 86, 523-534 (2016)

3. Lin, F.H., Wang, J.P., Zhou, X.W., Ma, W.X., et al.: Observation of interaction phenomena for two dimensionally reduced nonlinear models. Nonlinear Dyn. 94, 2643-2654 (2018)

4. Yin, Y.H., Ma, W.X., Liu, J.G., Lv, X.: Diversity of exact solutions to a $(3+1)$-dimensional nonlinear evolution equation and its reduction. Comput. Math. Appl. 76, 1275-1283 (2018)

5. Gao, X.Y.: Mathematical view with observational/experimental consideration on certain $(2+1)$-dimensional waves in the cosmic/laboratory dusty plasmas. Appl. Math. Lett. 91, 165-172 (2019)

6. Yuan, Y.Q., Tian, B., Liu, L., et al.: Solitons for the $(2+1)$-dimensional Konopelchenko-Dubrovsky equations. J. Math. Anal. Appl. 460, 476-486 (2018)

7. Liu, M.S., Li, X.Y., Zhao, Q.L.: Exact solutions to Euler equation and Navier-Stokes equation. Z. Angew. Math. Phys. 70(2), 1-13 (2019)

8. Bhrawy, A.H., Abdelkawy, M.A., Biswas, A.: Cnoidal and snoidal wave solutions to coupled nonlinear wave equations by the extended Jacobi's elliptic function method. Nonlinear Sci. Numer. Simul. 18, 915-925 (2013)

9. Ma, W.X., Lee, J.H.: A transformed rational function method and exact solutions to the $3+1$ dimensional Jimbo-Miwa equation. Chaos Solitons Fractals 42, 1356-1363 (2009)

10. Gao, L.N., Zi, Y.Y., Yin, Y.H., Ma, W.X., et al.: Bäcklund transformation, multiple wave solutions and lump solutions to a $(3+1)$-dimensional nonlinear evolution equation. Nonlinear Dyn. 89, 2233-2240 (2017)

11. Weiss, J.: The Painleve property for partial differential equations. Bäcklund transformation, Lax pairs, and the Schwarzian derivative. J. Math. Phys. 24, 1405-1413 (1983) 
12. Liu, L., Tian, B., Yuan, Y.Q., Du, Z.: Dark-bright solitons and semirational rogue waves for the coupled Sasa-Satsuma equations. Phys. Rev. E 97, 052217 (2018)

13. Ha, J.T., Zhang, H.Q., Zhao, Q.L.: Exact solutions for a Dirac-type equation with N-fold Darboux transformation. J. Appl. Anal. Comput. 9(1), 200-210 (2019)

14. Hirota, R.: The Direct Methods in Soliton Theory. Cambridge University, Cambridge (2004)

15. Ying, J.P., Lou, S.Y.: Multilinear variable separation approach in (3+1)-dimensions: the Burgers equation. Chin. Phys. Lett. 20, 1448-1451 (2003)

16. Wazwaz, A.M.: The Camassa-Holm-KP equations with compact and noncompact travelling wave solutions. Appl. Math. Comput. 170, 347-360 (2005)

17. Zhang, X.L., Zhang, H.Q.: A new generalized Riccati equation rational expansion method to a class of nonlinear evolution equation with nonlinear terms of any order. Appl. Math. Comput. 186, 705-714 (2007)

18. Cao, R., Zhang, J.: Trial function method and exact solutions to the generalized nonlinear Schrödinger equation with time-dependent coefficient. Chin. Phys. B 22, 100507 (2013)

19. Cai, K.J., Tian, B., Zhang, H., Meng, X.H.: Direct approach to construct the periodic wave solutions for two nonlinear evolution equations. Commun. Theor. Phys. 52, 473-478 (2009)

20. Ma, W.X., Zhou, R.G.: Exact one-periodic and two-periodic wave solutions to Hirota bilinear equations in $(2+1)$ dimensions. Mod. Phys. Lett. A 24(21), 1677-1688 (2009)

21. Tian, S.F., Zhang, H.Q.: Riemann theta functions periodic wave solutions and rational characteristics for the $(1+1)$-dimensional and (2+1)-dimensional ito equation. Chaos Solitons Fractals 47, $27-41$ (2013)

22. Wang, X.B., Tian, S.F., Xu, M.J., et al.: On integrability and quasi-periodic wave solutions to a (3+1)-dimensional generalized KdV-like model equation. Comput. Math. Appl. 283, 216-233 (2016)

23. Chen, Y.R., Liu, Z.R.: Riemann theta solutions and their asymptotic property for a $(3+1)$-dimensional water wave equation. Nonlinear Dyn. 87(2), 1069-1080 (2017)

24. Demiray, S., Tascan, F.: Quasi-periodic solutions of $(3+1)$ generalized BKP equation by using Riemann theta functions. Appl. Math. Comput. 273, 131-141 (2016)

25. Yu, S.J., Toda, K., Sasa, N., Fukuyama, T.: N-soliton solutions to the Bogoyavlenskii-Schiff equation and a quest for the soliton solution in (3 + 1) dimensions. Physica A 31, 3337-3347 (1998)

26. Ja'afar, A., Jawad, M., Petkovic, M.D.: Soliton solutions for nonlinear Calaogero-Degasperis and potential Kadomtsev-Petviashvili equations. Comput. Math. Appl. 62, 2621-2628 (2011)

\section{Submit your manuscript to a SpringerOpen ${ }^{\circ}$ journal and benefit from:}

- Convenient online submission

- Rigorous peer review

- Open access: articles freely available online

- High visibility within the field

- Retaining the copyright to your article

Submit your next manuscript at $\boldsymbol{\triangleright}$ springeropen.com 